\title{
Ceftobiprole: a potential empirical post-operative monotherapy in prosthetic joint infections
}

Claire Duployez ${ }^{1}$, Frédéric Wallet ${ }^{1}$, Henri Migaud ${ }^{2,4}$, Eric Senneville $^{3,4}$ and Caroline Loiez ${ }^{1 *}$

\begin{abstract}
Background: This study aimed to evaluate in vitro susceptibility to ceftobiprole of clinical strains identified from prosthetic joint infections (PJIs) compared to that of the associations currently recommended for post-operative empirical antibiotic therapy (PEAT) (vancomycin with either cefepime, third-generation cephalosporin or piperacillin-tazobactam).
\end{abstract}

Methods: We performed a 1-year retrospective study on all the surgical procedures performed in our hospital for PJI. Susceptibility profiles of all the strains cultured from surgical samples were reviewed to compare ceftobiprole to current used associations.

Results: During the study period (from January 2018 to December 2018), we identified 106 patients managed for $\mathrm{PJI}$ and a total of 216 surgical interventions. One hundred-fifty strains were identified from intraoperative samples, excluding redundant strains. Staphylococcus spp. represented $52.7 \%$ of all strains and Enterobacteriales $13.3 \%$. Twentythree patients had polymicrobial infection (22\%). Among 149 surgical procedures with positive culture results, ceftobiprole covered the bacterial strains in 138 (92.6\%) cases. In comparison, this percentage was $94.6 \%$ for vancomycin plus cefepime ( $p=0.64), 92.6 \%$ for vancomycin plus a third-generation cephalosporin in 138 cases $(p=1)$ and $94.6 \%$ for vancomycin plus piperacillin-tazobactam) $(p=0.64)$.

Conclusion: Based on antimicrobial susceptibility testing, our results suggest that ceftobiprole could be an interesting option for PEAT in PJls, allowing the use of a single agent.

Keywords: Prosthetic joint infection, Ceftobiprole, Empirical antibiotic therapy, Bacterial resistance

\section{Background}

Prosthetic joint infections (PJI) require both surgical intervention and antibiotic therapy conducted according to the most recent guidelines for the management of these potentially life-threatening infections $[1,2]$.

The results of the susceptibility profile of the bacteria isolated from the intraoperative samples usually require at least 5 days to become available. During this period, an initial post-operative empirical antibiotic therapy (PEAT)

\footnotetext{
*Correspondence: caroline.loiez@chru-lille.fr

${ }^{1}$ Institute of Microbiology, Lille University Hospital, 59037 Lille, France

Full list of author information is available at the end of the article
}

is usually administered to prevent the colonization of newly prosthesis or the prosthesis that has been cleaned but has been retained during the so-called debridementantibiotic and implant retention (DAIR). Given the important role of staphylococci in PJI, the PEAT needs to be active against most Gram-positive cocci including methicillin-resistant staphylococci but also Gram-negative bacilli. Currently, there has been no consensus on the optimal choice of this antibiotic therapy. In France, the combinations of vancomycin combined with either a third-generation cephalosporin or piperacillin-tazobactam are proposed choices [3].

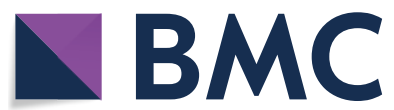

c The Author(s) 2020. This article is licensed under a Creative Commons Attribution 4.0 International License, which permits use, sharing, adaptation, distribution and reproduction in any medium or format, as long as you give appropriate credit to the original author(s) and the source, provide a link to the Creative Commons licence, and indicate if changes were made. The images or other third party material in this article are included in the article's Creative Commons licence, unless indicated otherwise in a credit line to the material. If material is not included in the article's Creative Commons licence and your intended use is not permitted by statutory regulation or exceeds the permitted use, you will need to obtain permission directly from the copyright holder. To view a copy of this licence, visit http://creativeco mmons.org/licenses/by/4.0/. The Creative Commons Public Domain Dedication waiver (http://creativecommons.org/publicdomain/ zero/1.0/) applies to the data made available in this article, unless otherwise stated in a credit line to the data. 
Ceftobiprole is a newly commercialized beta-lactam antibiotic with a broad spectrum, equivalent to an association of a third-generation cephalosporin plus vancomycin. Notably, it is active against methicillin-resistant staphylococci, Enterococcus faecalis, most extended spectrum beta-lactamase-non-producing Enterobacteriales and Pseudomonas aeruginosa. It may replace use of this associations for PEAT.

The main objective of the present study was to assess the antibacterial in vitro activity of ceftobiprole on strains recovered from PJI infections in order to enable or not its use as a single molecule in PEAT. We therefore compared ceftobiprole to vancomycin plus cefepime, vancomycin plus third generation cephalosporin (e.g. cefotaxime or ceftriaxone) and vancomycin plus piperacillin-tazobactam in this setting.

\section{Methods}

\section{Definitions}

PJIs were identified according to the Infectious Diseases Society of America definition [2].

\section{Study design and population}

This retrospective study was performed at the French National Reference Centre for Complex Osteoarticular Infections in the North West region of France (Roger Salengro Hospital, Lille, France). Medical charts of all adult patients with documented PJI who received PEAT from January 2018 to December 2018 were reviewed. All patients included in the study had surgical management including DAIR, one or two-step implant exchange.

\section{Surgical management and curative antibiotic therapy}

All surgical procedures (i.e., implant retention, or oneto two-step implant exchange) were performed without antibiotic prophylaxis. Ceftobiprole administered intravenously was begun intraoperatively immediately after samples were taken. PEAT was continued until the results of intraoperative sample cultures were available and was then modified in accordance with the culture results (curative antibiotic therapy). Therapeutic strategies were decided for each patient during multidisciplinary meetings of orthopaedic surgeons, infectious diseases consultants, microbiologists and anaesthesiologists, based on the patient's characteristics and were administered following the recommendations of Zimmerli et al. [4]. In each case, the patient was aware of the different therapeutic options and took part in the final decision.

\section{Microbiology}

During surgical procedures, at least three tissue samples were taken in different areas suspected of being affected using a separate sterile instrument for each sample. Three
$\mathrm{mL}$ of sterile saline were added to each sample, and samples were vigorously shaked during $1 \mathrm{~min}$ using sterile glass beads, in order to disrupt tissue and release bacteria. Then, one drop was inoculated onto a Columbia agar with blood $5 \%$ (incubation at $37{ }^{\circ} \mathrm{C}$ in air for 5 days), one drop onto a chocolate agar with polyvitex (incubation at $37{ }^{\circ} \mathrm{C}$ in $\mathrm{CO}_{2}$ for 5 days) and one-millilitre aliquots were inoculated into an Aerobic VIRTUO blood culture bottle, and an anaerobic VIRTUO blood culture bottle (BioMérieux, Marcy l'Etoile, France). All plates were examined daily for 5 days. VIRTUO blood culture bottles were placed on the VIRTUO system for 14 days and were subcultured if they flagged positive." Strains were identified using MALDI-TOF spectrometry mass (Bruker Daltonics, Wissembourg, France) with a minimal score requirement of 2 .

The antibiotic susceptibility profile of all pathogens identified from intraoperative samples was assessed either by the Vitek 2 cards (BioMérieux, Marcy l'Etoile, France) or by agar diffusion technique using the procedure and interpretation criteria proposed by the Comité de l'Antibiogramme de la Société Française de Microbiologie (CA-SFM EUCAST 2018) (http://www.sfmmicrobiologie.org). Minimum inhibitory concentrations (MIC) of ceftobiprole were determined with the Etest (Liofilchem, Roseto degli Abruzzi, Italy). Methicillin resistance was confirmed by detection of $m e c A$ gene if required.

\section{Results \\ Patients}

During the study period, we identified 106 patients managed for PJI (57 hip prosthesis, 39 knee prosthesis, 9 shoulder prosthesis, 4 elbow prosthesis and 4 other prosthesis, with sometimes more than one prosthesis by patient). A total of 216 surgical interventions were identified from these 106 patients. Among these 216 surgical interventions, 67 remained negative for the three samples (31\%).The demographic characteristics of the included patients are reported in Table 1.

\section{Microbiology}

A total of 150 clinical strains were identified from intraoperative samples, excluding redundant strains taken in different surgical interventions performed on the same patient (Table 2).

Staphylococcus spp. accounted for $52.7 \%$ of all strains, especially coagulase negative staphylococci (CoNS) $(32.7 \%$ of all strains) with $53.1 \%$ of methicillin-resistant and $20.4 \%$ of teicoplanin-resistant strains. S. aureus accounted for $20.0 \%$ of all strains including 2 methicillin-resistant S. aureus (MRSA). Streptococcus spp. and Enterococcus spp. accounted for $21.3 \%$ of all strains. 
Table 1 Characteristics of 106 patients (216 events) with PJI

\begin{tabular}{|c|c|}
\hline \multicolumn{2}{|l|}{ Characteristics } \\
\hline Male, N of patients (\%) & $53(50)$ \\
\hline Female, $\mathrm{N}$ of patients (\%) & $53(50)$ \\
\hline Age, years, mean $\pm S D$ (range) & $67 \pm 12.9(26-95)$ \\
\hline$\geq 75(\%)$ & $27(25.5)$ \\
\hline $50-75(\%)$ & $70(66)$ \\
\hline$<50(\%)$ & $9(8.5)$ \\
\hline \multicolumn{2}{|l|}{ Location of PJI, N of patients (\%) } \\
\hline Hip & 57 \\
\hline Knee & 39 \\
\hline Shoulder & 9 \\
\hline Elbow & 4 \\
\hline Other prosthesis & 4 \\
\hline $\begin{array}{l}\text { Fever at the time of surgical management, } N \text { of } \\
\text { events (\%) (data available for } 214 \text { events) }\end{array}$ & $14(6.5 \%)$ \\
\hline \multicolumn{2}{|c|}{ Biological characteristics at the time of surgical management } \\
\hline $\begin{array}{l}\text { CRP (mg/L) } \pm S D \text { (range) (data available for } \\
164 \text { events) }\end{array}$ & $76.64 \pm 91.9(<3-431)$ \\
\hline $\begin{array}{l}\text { Leucocytes }\left(\times 10^{9} / \mathrm{L}\right) \pm \mathrm{SD} \text { (range) (data avail- } \\
\text { able for } 177 \text { events) }\end{array}$ & $9.35 \pm 3.51(4.30-29.42)$ \\
\hline $\begin{array}{l}\text { Creatinine (mg/L) } \pm S D \text { (range) (data available } \\
\text { for } 178 \text { events) }\end{array}$ & $8.60 \pm 3.76(3-29)$ \\
\hline
\end{tabular}

$S D$ standard deviation

Enterobacteriales represented $13.3 \%$ of all strains including 4 extended-spectrum beta-lactamases (ESBL)producing Enterobacteriales. Among non-fermenting Gram-negative bacilli, 3 strains of Pseudomonas aeruginosa and 1 strain of Acinetobacter baumannii were identified. At last, for 2 patients, yeasts were identified. Twenty-three patients had polymicrobial infection (22\%).

We evaluated the in vitro susceptibility to ceftobiprole, and to the associations of vancomycin with either cefepime, a third-generation cephalosporin (cefotaxime, ceftriaxone) or piperacillin-tazobactam. Results are presented in Table 2 and resistant strains to at least one of these therapies are detailed in Table 3. Among Gram-positive cocci, all strains were susceptible to the 3 associations and all but the strain of E. raffinosus were susceptible to ceftobiprole. Overall, $100 \%$ of $S$. aureus and CoNS strains were inhibited at the CASFM EUCAST breakpoints of $2 \mathrm{mg} / \mathrm{L}$ (breakpoint for $S$. aureus) and $4 \mathrm{mg} / \mathrm{L}$ (non-species-related breakpoint), respectively. $\left(\mathrm{MIC}_{50 / 90}\right.$ of $0.5 / 0.75$ and $0.5 / 1$ respectively). Only one methicillin-resistant $S$. haemolyticus isolate had a MIC of $4 \mathrm{mg} / \mathrm{L}$. For Enterobacteriales, 4 ESBL-producing strains were resistant to ceftobiprole as to the association including cefepime or a third-generation cephalosporin (patients C, D, G, H). Piperacillin-tazobactam was effective against one of these ESBL-producing strains (patient $C$ ). The $3 P$. aeruginosa strains were susceptible to cefepime and piperacillin-tazobactam; one was resistant to ceftobiprole. OXA23-producing A. baumannii was resistant to the 4 therapies evaluated. Due to their spectrum of activity, none of these therapies was effective on the 2 yeasts and none except piperacillin-tazobactam was effective on B. thetaiotaomicron.

Finally, over the 216 reported surgical procedures, microorganisms were cultured for 149 of them. Based on antimicrobial susceptibility testing, ceftobiprole could have been used with success in 138 cases among these 149 interventions, versus vancomycin plus cefepime in 141 cases $(p=0.64)$, vancomycin plus a third-generation cephalosporin in 138 cases $(\mathrm{p}=1)$ and vancomycin plus piperacillin-tazobactam in 141 cases $(p=0.64)$. Empirical use of ceftobiprole would have been ineffective in 8 patients (patients A, B, C, D, G, H, I, K) (Table 3; Fig. 1).

\section{Discussion}

Ceftobiprole is currently not approved for the treatment of PJIs but its broad spectrum of activity including most of the bacteria responsible for these infections makes it an attractive candidate for empirical antibiotic therapy following surgical septic revision of PJIs. Our results suggest that ceftobiprole would have been effective on $92.6 \%$ of surgical procedures. Our epidemiology being like that found in the literature [4], ceftobiprole is non inferior compared to the three associations evaluated: vancomycin plus cefepime, a third-generation cephalosporin or piperacillin-tazobactam, in terms of microbial susceptibility. The good correlation between in vitro and in vivo activity of ceftobiprole has been confirmed [5] as well as the low potential for emergence of resistance under treatment [6].

Ceftobiprole is active against most of Gram-positive cocci, including staphylococci, which are the most prevalent bacteria identified in our patients, accounting for $74 \%$ of the microorganisms cultured). All are susceptible in vitro to ceftobiprole with $\mathrm{MIC}_{50 / 90}$ of $0.5 / 1$ and one strain of CoNS with a MIC of $4 \mathrm{mg} / \mathrm{L}$. These results are like those obtained by Isnard et al. [7] in a study performed on $100 \mathrm{~S}$. aureus strains and 100 CoNS strains cultured from PJIs: all but 2 S. aureus strains (with MIC of $4 \mathrm{mg} / \mathrm{L}$ ) had MIC lower than $2 \mathrm{mg} / \mathrm{L}$. In another series of 33 strains of S. epidermidis, including one-third multiresistant strains, Hellmark et al. [8] found $\mathrm{MIC}_{50}$ and $\mathrm{MIC}_{90}$ values of 0.5 and $1.5 \mathrm{mg} / \mathrm{L}$ respectively.

In our study, $53.1 \%$ of CoNS and $6.6 \%$ of $S$. aureus were methicillin-resistant strains and all were susceptible to ceftobiprole. In the literature, Rouse et al. studied the activity of ceftobiprole against MRSA (31 isolates) and CoNS (65 isolates) from bone and joint infections: all strains had MIC $\leq 2 \mathrm{mg} / \mathrm{L}$ (except for one CoNS with 
Table 2 Pathogens isolated from intraoperative samples of PJI in 106 patients and their susceptibility profile to the antimicrobial therapy evaluated

\begin{tabular}{|c|c|c|c|c|c|}
\hline Microorganism & $\begin{array}{l}\text { Number } \\
\text { of strains } \\
\text { (\% of the total) }\end{array}$ & $\begin{array}{l}\text { Ceftobiprole: } \\
\text { resistant } \\
\text { strains }\end{array}$ & $\begin{array}{l}\text { Vancomycine + } \\
\text { cefepime: } \\
\text { resistant } \\
\text { strains }\end{array}$ & $\begin{array}{l}\text { Vancomycine + } \\
\text { 3rd generation } \\
\text { cephalosporin: } \\
\text { resistant strains }\end{array}$ & $\begin{array}{l}\text { Vancomycine + } \\
\text { piperacillin- } \\
\text { tazobactam: } \\
\text { resistant strains }\end{array}$ \\
\hline Gram-positive cocci & $111(74.0)$ & 1 & 0 & 0 & 0 \\
\hline Staphylococci & $79(52.7)$ & - & - & - & - \\
\hline Staphylococcus aureus & $30(20.0)$ & - & - & - & - \\
\hline MSSA & $28(18.7)$ & - & - & - & - \\
\hline MRSA & $2(1.3)$ & - & - & - & - \\
\hline CoNS & $49(32.7)$ & - & - & - & - \\
\hline Staphylococcus epidermidis & $30(20.0)$ & - & - & - & - \\
\hline Staphylococcus capitis & $7(4.7)$ & - & - & - & - \\
\hline Staphylococcus caprae & $4(2.7)$ & - & - & - & - \\
\hline Staphylococcus haemolyticus & $1(0.7)$ & - & - & - & - \\
\hline Staphylococcus lugdunensis & $4(2.7)$ & - & - & - & - \\
\hline Staphylococcus pettenkoferi & $2(1.3)$ & - & - & - & - \\
\hline Staphylococcus warneri & $1(0.7)$ & - & - & - & - \\
\hline Streptococcus spp. & $20(13.3)$ & - & - & - & - \\
\hline Streptococcus adiacens & $1(0.7)$ & - & - & - & - \\
\hline Streptococcus anginosus & $1(0.7)$ & - & - & - & - \\
\hline Streptococcus agalactiae & $6(4.0)$ & - & - & - & - \\
\hline Streptococcus dysgalactiae & $3(2.0)$ & - & - & - & - \\
\hline Streptococcus gallolyticus & $1(0.7)$ & - & - & - & - \\
\hline Streptococcus gordonii & $2(1.3)$ & - & - & - & - \\
\hline Streptococcus mitis/oralis & $2(1.3)$ & - & - & - & - \\
\hline Streptococcus pneumoniae & $1(0.7)$ & - & - & - & - \\
\hline Streptococcus parasanguinis & $1(0.7)$ & - & - & - & - \\
\hline Streptococcus sanguinis & $1(0.7)$ & - & - & - & - \\
\hline Streptococcus vestibularis & $1(0.7)$ & - & - & - & - \\
\hline Enterococci & $12(8.0)$ & 1 & - & - & - \\
\hline Enterococcus faecalis & $11(7.3)$ & - & - & - & - \\
\hline Enterococcus raffinosus & $1(0.7)$ & 1 & - & - & - \\
\hline Gram-negative bacilli & $24(16.0)$ & 6 & 5 & 8 & 4 \\
\hline Enterobacteriales & $20(13.3)$ & 4 & 4 & 4 & 3 \\
\hline Citrobacter freundii & $1(0.7)$ & - & - & - & - \\
\hline Citrobacter koseri & $1(0.7)$ & - & - & - & - \\
\hline Enterobacter cloacae (2 ESBL-producing strain) & $3(2.0)$ & 2 & 2 & 2 & 2 \\
\hline Escherichia coli (1 ESBL-producing strain) & $4(2.7)$ & 1 & 1 & 1 & 1 \\
\hline Klebsiella oxytoca & $1(0.7)$ & - & - & - & - \\
\hline Klebsiella pneumoniae (1 ESBL-producing strain) & $4(2.7)$ & 1 & 1 & 1 & 2 \\
\hline Morganella morganii & $1(0.7)$ & - & - & - & - \\
\hline Proteus mirabilis & $4(2.7)$ & - & - & - & - \\
\hline Serratia marcescens & $1(0.7)$ & - & - & - & - \\
\hline Non-fermenting bacilli & $4(2.7)$ & 2 & 1 & 4 & 1 \\
\hline Pseudomonas aeruginosa & $3(2.0)$ & 1 & - & 3 & - \\
\hline Acinetobacter baumannii (OXA23-producing strain) & $1(0.7)$ & 1 & 1 & 1 & 1 \\
\hline Anaerobes & $8(5.3)$ & 1 & 1 & 1 & 0 \\
\hline Cutibacterium acnes & $5(3.3)$ & - & - & - & - \\
\hline Cutibacterium avidum & $1(0.7)$ & - & - & - & - \\
\hline
\end{tabular}


Table 2 (continued)

\begin{tabular}{|c|c|c|c|c|c|}
\hline Microorganism & $\begin{array}{l}\text { Number } \\
\text { of strains } \\
\text { (\% of the total) }\end{array}$ & $\begin{array}{l}\text { Ceftobiprole: } \\
\text { resistant } \\
\text { strains }\end{array}$ & $\begin{array}{l}\text { Vancomycine + } \\
\text { cefepime: } \\
\text { resistant } \\
\text { strains }\end{array}$ & $\begin{array}{l}\text { Vancomycine+ } \\
\text { 3rd generation } \\
\text { cephalosporin: } \\
\text { resistant strains }\end{array}$ & $\begin{array}{l}\text { Vancomycine + } \\
\text { piperacillin- } \\
\text { tazobactam: } \\
\text { resistant strains }\end{array}$ \\
\hline Clostridium perfringens & $1(0.7)$ & - & - & - & - \\
\hline Bacteroides thetaiotaomicron & $1(0.7)$ & 1 & 1 & 1 & - \\
\hline Others & $7(4.7)$ & 2 & 2 & 2 & 2 \\
\hline Bacillus cereus & $1(0.7)$ & - & - & - & - \\
\hline Bacillus pumilus & $1(0.7)$ & - & - & - & - \\
\hline Corynebacterium striatum & $1(0.7)$ & - & - & - & - \\
\hline Micrococcus luteus & $2(1.3)$ & - & - & - & - \\
\hline Yeast & $2(1.3)$ & 2 & 2 & 2 & 2 \\
\hline Total number of strains & $150(100)$ & 10 & 8 & 11 & 6 \\
\hline
\end{tabular}

MIC value of $4 \mathrm{mg} / \mathrm{L}$ ) [9]. Our study also included 20.4\% of teicoplanin-resistant CoNS, all remaining susceptible to ceftobiprole; these results are consistent with those obtained by Henriksen et al. [10] on 136 teicoplaninresistant bacteremia CoNS strains (representing 20.9\% of their 650 bacteremia CoNS strains). Concerning vancomycin-resistant strains, Farrell et al. [11] demonstrated a potential high activity of ceftobiprole against 44 VISA and hVISA strains $\left(\mathrm{MIC}_{90}\right.$ of $\left.2 \mathrm{mg} / \mathrm{L}\right)$ and against 10 VRSA strains $\left(\mathrm{MIC}_{90}\right.$ of $\left.1 \mathrm{mg} / \mathrm{L}\right)$.

In addition, the bactericidal activity of ceftobiprole against Staphylococci has been proved in several studies: Isnard et al. [7] showed a rapid killing effect, with a significant and sustained decrease of the inoculum after a $24 \mathrm{~h}$ incubation period at concentration of 4* MIC while a phenomenon of re-growth was observed with vancomycin. Rouse et al. demonstrated it against 31 methicillin-resistant $S$. aureus and 65 CoNS isolated from bone and joint infections (all strains had MIC and MBC values $\leq 2 \mu \mathrm{g} / \mathrm{mL}$, except for one CoNS with MIC and $\mathrm{MBC}$ values of 4 and $8 \mu \mathrm{g} / \mathrm{mL}$ ) while vancomycin lacked bactericidal activity against 2 MRSA [9]. In vivo studies were also performed with ceftobiprole. For methicillin-susceptible strains, vancomycin is known to

Table 3 Susceptibility profile of the 16 strains resistant to at least 1 of the antimicrobial therapy evaluated

\begin{tabular}{|c|c|c|c|c|c|c|}
\hline Patient & $\begin{array}{l}\text { Surgical } \\
\text { procedure }\end{array}$ & Strains & Ceftobiprole & $\begin{array}{l}\text { Vancomycin- } \\
\text { cefepime }\end{array}$ & $\begin{array}{l}\text { Vancomycin- } \\
\text { 3rd generation } \\
\text { cephalosporin }\end{array}$ & $\begin{array}{l}\text { Vancomycin- } \\
\text { piperacillin- } \\
\text { tazobactam }\end{array}$ \\
\hline A & 1 & Yeast & $\mathrm{R}$ & $\mathrm{R}$ & $\mathrm{R}$ & $\mathrm{R}$ \\
\hline B & 1 & Yeast & $\mathrm{R}$ & $\mathrm{R}$ & $\mathrm{R}$ & $\mathrm{R}$ \\
\hline \multirow[t]{3}{*}{$C$} & 1 & E. raffinosus & $\mathrm{R}$ & $\mathrm{S}$ & S & S \\
\hline & 2 & E. raffinosus & $\mathrm{R}$ & $\mathrm{S}$ & S & S \\
\hline & 3 & ESBL-producing E. coli & $\mathrm{R}$ & $\mathrm{R}$ & $\mathrm{R}$ & $\mathrm{S}$ \\
\hline \multirow[t]{2}{*}{$\mathrm{D}$} & 1 & ESBL-producing E. cloacae & $\mathrm{R}$ & $\mathrm{R}$ & $\mathrm{R}$ & $\mathrm{R}$ \\
\hline & 2 & ESBL-producing E. cloacae & $\mathrm{R}$ & $\mathrm{R}$ & $\mathrm{R}$ & $\mathrm{R}$ \\
\hline $\mathrm{E}$ & 1 & E. coli & $\mathrm{s}$ & $\mathrm{S}$ & S & $\mathrm{R}$ \\
\hline $\mathrm{F}$ & 1 & K.pneumoniae & $\mathrm{S}$ & S & S & $\mathrm{R}$ \\
\hline \multirow[t]{2}{*}{ G } & 1 & ESBL-producing K. pneumoniae & $\mathrm{R}$ & $\mathrm{R}$ & $\mathrm{R}$ & $\mathrm{R}$ \\
\hline & & OXA23-producing A. baumannii & $\mathrm{R}$ & $\mathrm{R}$ & $\mathrm{R}$ & $\mathrm{R}$ \\
\hline \multirow[t]{2}{*}{$\mathrm{H}$} & 1 & ESBL-producing E. cloacae & $\mathrm{R}$ & $\mathrm{R}$ & $\mathrm{R}$ & $\mathrm{R}$ \\
\hline & 2 & P. aeruginosa & $\mathrm{s}$ & $\mathrm{S}$ & $\mathrm{R}$ & $\mathrm{S}$ \\
\hline । & 1 & P. aeruginosa & $\mathrm{R}$ & $\mathrm{S}$ & $\mathrm{R}$ & $\mathrm{s}$ \\
\hline J & 1 & P. aeruginosa & $\mathrm{s}$ & $\mathrm{S}$ & $\mathrm{R}$ & $\mathrm{S}$ \\
\hline K & 1 & B. thetaiotaomicron & $\mathrm{R}$ & $\mathrm{R}$ & R & $\mathrm{S}$ \\
\hline
\end{tabular}




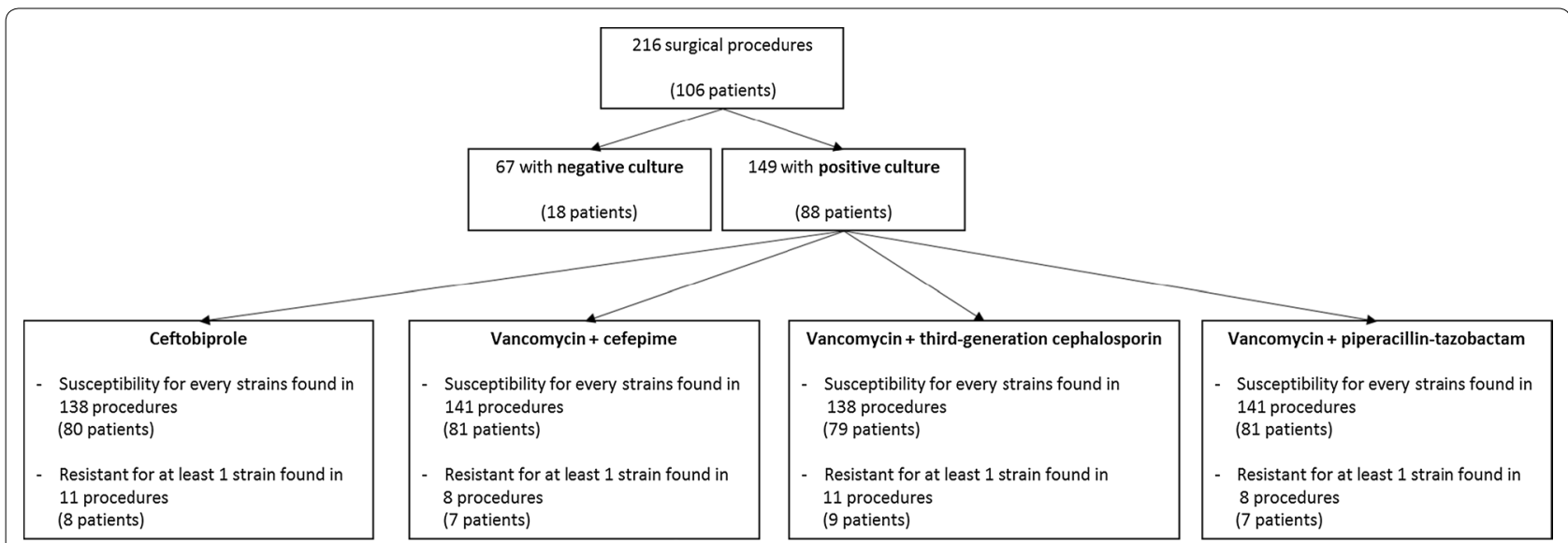

Fig. 1 Antimicrobial susceptibility for the 216 surgical procedures

have a lesser efficiency against methicillin-susceptible strains compared with beta-lactams and ceftobiprole was superior to ceftriaxone and vancomycin in a mouse experimental septicemia model [5]. In vivo activity of ceftobiprole was also shown in a MRSA endocarditis model in rabbits [12] and in VISA infection models [5].

PJI may also be caused by streptococci, and Gramnegative bacilli on which ceftobiprole maintains the activity of extended-spectrum cephalosporins. In our study including 20 Enterobacteriales strains, a resistance to ceftobiprole was detected only for the 4 ESBL-producing strains, which were also resistant to cefepime and third-generation cephalosporins.

For anaerobic bacteria, it has a similar spectrum as amoxicillin plus clavulanic acid. It is active against $P$. aeruginosa strains, mostly in ceftazidime-susceptible strains but has a poor activity on A. baumannii [13, 14].

The main insufficiency of ceftobiprole in the setting of PEAT for PJIs are (i) ampicillin-resistant strains of E. faecium (on which vancomycin may be effective), (ii) strains of B. fragilis group species, and (iii) ESBLproducing Enterobacteriales (on which only piperacillin-tazobactam may be effective). These strains are not frequently found as causative agents of PJI and infections with ESBL-producing Enterobacteriales may be suspected on the basis of the patients' medical history. Thus, and as for any antibiotic therapy, bacteriological history of the patient and microbial ecology of the hospital must be considered when choosing the PEAT.

Our study presents some limitations. First, one-third of the total specimens remained sterile. This may seem important, but we included surgical interventions in patients previously infected in order to control the sterility of the specimens. Furthertheless, it does not evaluate activity on microorganisms that grow in biofilms, which cannot be evaluated routinely. Biofilm impairs the activity of most antibiotics by reducing their accessibility to bacteria. Moreover, bacteria in biofilms are for some of them in a stationary phase [4] which results in an increased resistance to most antibiotics, especially those with a growth-dependent mode of action such as ceftobiprole. Nevertheless, an in vitro study performed by Abbanat et al. [15] showed a CFU decrease $>2 \log _{10}$ on immature and mature staphylococcal biofilms by ceftobiprole which seems to be less affected by biofilm age than vancomycin. Their results are consistent with in vivo results obtained in endocarditis infection model [12]. For treatment of PJI, antibiotics used must diffuse in the bone tissue. Yin et al. [16] studied pharmacokinetics and distribution into bone tissue of ceftobiprole in a rabbit model of osteomyelitis, pointing out promising results with bacterial titers in infected tibiae below the level of detection after treatment with ceftobiprole (versus $73 \%$ of the infected tibiae treated by vancomycin) and mean titers of the molecule 3 to 5 times higher in infected bones than in uninfected bones. Clinical data, however, are lacking. It may be useful to use a single molecule rather than an association in terms of adverse effects of the therapy.

Our results suggest that ceftobiprole used as PEAT for PJIs shows similar spectrum of activity as the associations vancomycin plus a beta-lactam currently recommended and could be an attractive alternative to the combination antibiotic therapy currently proposed.

\section{Acknowledgements \\ Not applicable.}

\section{Authors' contributions}

$C D, F W$ and $C L$ collected the data and analyzed the results, supervised the work, and wrote the article. ES and HM managed the patients, and contributed to reviewing the article. All authors read and approved the final manuscript. 


\section{Funding}

None to declare

\section{Availability of data and materials}

Not applicable to data. All information utilized could be found using the references provided in body of the manuscript.

\section{Ethics approval and consent to participate}

Not applicable.

\section{Consent for publication}

Not applicable.

\section{Competing interests}

The authors declare that they have no competing interests.

\section{Author details}

${ }^{1}$ Institute of Microbiology, Lille University Hospital, 59037 Lille, France.

${ }^{2}$ Orthopaedic Department, Lille University Hospital, 59037 Lille, France. ${ }^{3}$ Infectious Diseases Department, Gustave Dron Hospital, 59200 Tourcoing, France.

${ }^{4}$ University Hospital of Lille, 59037 Lille, France.

Received: 5 September 2019 Accepted: 12 March 2020

Published online: 21 March 2020

\section{References}

1. Parvizi J, Gehrke T, Chen AF. Proceedings of the international consensus on periprosthetic joint infection. Bone Joint J. 2013;95-B(11):1450-2. https ://doi.org/10.1302/0301-620x.95b11.33135.

2. Osmon DR, Berbari EF, Berendt AR, Lew D, Zimmerli W, Steckelberg JM, Rao N, Hanssen A, Wilson WR, Infectious Diseases Society of America. Diagnosis and management of prosthetic joint infection: clinical practice guidelines by the Infectious Diseases Society of America. Clin Infect Dis. 2013;56(1):e1-25. https://doi.org/10.1093/cid/cis803.

3. Haute Autorité de Santé. Recommandations de bonne pratiqueProthèse de hanche ou de genou: diagnostic et prise en charge de I'infection dans le mois suivant l'implantation. 2014. www.has-sante.fr.

4. Zimmerli W, Trampuz A, Ochsner PE. Prosthetic-joint infections. N Engl J Med. 2004;351(16):1645-54.

5. Chambers HF. Ceftobiprole: in vivo profile of a bactericidal cephalosporin. Clin Microbiol Infect. 2006;12(Suppl 2):17-22 (Review).

6. Barbour A, Schmidt S, Rand KH, Derendorf H. Ceftobiprole: a novel cephalosporin with activity against Gram-positive and Gram-negative pathogens, including methicillin-resistant Staphylococcus aureus (MRSA) Int J Antimicrob Agents. 2009;34(1):1-7. https://doi.org/10.1016/j.ijant imicag.2008.12.012.

7. Isnard C, Dhalluin A, Malandain D, Bruey Q, Auzou M, Michon J, Giard JC, Guérin F, Cattoir V. In vitro activity of novel anti-MRSA cephalosporins and comparator antimicrobial agents against staphylococci involved in prosthetic joint infections. J Glob Antimicrob Resist. 2018;13:221-5. https ://doi.org/10.1016/j.jgar.2018.01.022.

8. Hellmark B, Unemo M, Nilsdotter-Augustinsson A, Söderquist B. Antibiotic susceptibility among Staphylococcus epidermidis isolated from prosthetic joint infections with special focus on rifampicin and variability of the rpoB gene. Clin Microbiol Infect. 2009;15(3):238-44. https://doi.org/10.111 1/j.1469-0691.2008.02663.x.

9. Rouse MS, Steckelberg JM, Patel R. In vitro activity of ceftobiprole, daptomycin, linezolid, and vancomycin against methicillin-resistant staphylococci associated with endocarditis and bone and joint infection. Diagn Microbiol Infect Dis. 2007;58(3):363-5.

10. Henriksen AS, Smart J, Hamed K. Comparative activity of ceftobiprole against coagulase-negative staphylococci from the BSAC Bacteraemia Surveillance Programme, 2013-2015. Eur J Clin Microbiol Infect Dis. 2018;37(9):1653-9. https://doi.org/10.1007/s1 0096-018-3295-6.

11. Farrell DJ, Flamm RK, Sader HS, Jones RN. Activity of ceftobiprole against methicillin-resistant Staphylococcus aureus strains with reduced susceptibility to daptomycin, linezolid or vancomycin, and strains with defined SCCmec types. Int J Antimicrob Agents. 2014;43(4):323-7. https://doi. org/10.1016/j.jjantimicag.2013.11.005.

12. Tattevin P, Basuino L, Bauer D, Diep BA, Chambers HF. Ceftobiprole is superior to vancomycin, daptomycin, and linezolid for treatment of experimental endocarditis in rabbits caused by methicillin-resistant Staphylococcus aureus. Antimicrob Agents Chemother. 2010;54(2):610-3. https://doi.org/10.1128/AAC.00886-09.

13. Farrell DJ, Flamm RK, Sader HS, Jones RN. Ceftobiprole activity against over 60,000 clinical bacterial pathogens isolated in Europe, Turkey, and Israel from 2005 to 2010. Antimicrob Agents Chemother. 2014;58(7):3882-8. https://doi.org/10.1128/AAC.02465-14.

14. Pfaller MA, Flamm RK, Duncan LR, Streit JM, Castanheira M, Sader HS. Antimicrobial activity of ceftobiprole and comparator agents when tested against contemporary Gram-positive and -negative organisms collected from Europe (2015). Diagn Microbiol Infect Dis. 2018;91(1):77-84. https://doi.org/10.1016/j.diagmicrobio.2017.12.020.

15. Abbanat D, Shang W, Amsler K, Santoro C, Baum E, Crespo-Carbone S, Lynch AS. Evaluation of the in vitro activities of ceftobiprole and comparators in staphylococcal colony or microtitre plate biofilm assays. Int J Antimicrob Agents. 2014;43(1):32-9. https://doi.org/10.1016/j.jiantimica g.2013.09.013.

16. Yin LY, Calhoun JH, Thomas JK, Shapiro S, Schmitt-Hoffmann A. Efficacies of ceftobiprole medocaril and comparators in a rabbit model of osteomyelitis due to methicillin-resistant Staphylococcus aureus. Antimicrob Agents Chemother. 2008:52(5):1618-22. https://doi.org/10.1128/ AAC.00638-07.

\section{Publisher's Note}

Springer Nature remains neutral with regard to jurisdictional claims in published maps and institutional affiliations.
Ready to submit your research? Choose BMC and benefit from:

- fast, convenient online submission

- thorough peer review by experienced researchers in your field

- rapid publication on acceptance

- support for research data, including large and complex data types

- gold Open Access which fosters wider collaboration and increased citations

- maximum visibility for your research: over $100 \mathrm{M}$ website views per year

At BMC, research is always in progress.

Learn more biomedcentral.com/submissions 